\title{
Incomplete Information and Rent Dissipation in Deterministic Contests*
}

\author{
René Kirkegaard \\ Department of Economics \\ University of Guelph \\ rkirkega@uoguelph.ca
}

August 2010

\begin{abstract}
In a deterministic contest or all-pay auction, all rents are dissipated when information is complete and contestants are identical. As one contestant becomes "stronger", that is, values the prize more, total expenditures are known to decrease monotonically. Thus, asymmetry among contestants reduces competition and rent dissipation. Recently, this result has been shown to hold for other, non-deterministic, contest success functions as well, thereby suggesting a certain robustness. In this paper, however, the complete information assumption is shown to be crucial. With incomplete information - regardless of how little - total expenditures in a deterministic two-player contest increase when one contestant becomes marginally stronger, starting from a symmetric contest. In fact, both contestants expend resources more aggressively; with complete information, neither of them do so.
\end{abstract}

JEL Classification Numbers: C72, D44, D72, D82.

Keywords: All-pay auctions, Asymmetric auctions, Rent seeking.

${ }^{*}$ I would like to thank the Canada Research Chairs programme and the Social Sciences and Humanities Research Council of Canada for funding this research. I am grateful to J. Atsu Amegashie for detailed comments on an earlier draft. 


\section{Introduction}

Tullock's (1967, 1980) famous pair of papers have triggered a large literature on rent seeking. In large part, the focus of this literature is on the wasteful use of resources to contest rents. Applications thus include war, lobbying, and campaign spending, to mention but a few. Usually, complete information is assumed. That is, the value that each contestant place on winning the prize is assumed to be common knowledge. ${ }^{1}$ Simply put, the objective of the current paper is to make the point that some of the important findings of this literature are not robust to the inclusion of incomplete information. Indeed, in some respects the comparative statics of contests with incomplete information are precisely the opposite of the comparative statics that are known from contests with complete information.

Consider a deterministic contest, or all-pay auction. In such a contest, the contestant who expends the most resources wins the prize with probability one. Assume there are two contestants, and that contestant $i$ is known to value the prize at $v_{i}$, $i=1,2$. These rules and payoffs define a complete information game. The game has a unique Nash equilibrium in mixed strategies; contestants randomize to keep the opposition guessing. See Hillman and Riley (1989) and Baye, Kovenock, and de Vries (1996) for details. If $v_{1} \geq v_{2}$, the expected expenditures of contestants 1 and 2 are, respectively,

$$
E P_{1}^{c}=\frac{v_{2}}{2} \text { and } E P_{2}^{c}=\frac{v_{2}^{2}}{2 v_{1}}
$$

If the contest is symmetric, or $v_{1}=v_{2}$, then all rents are dissipated since $E P_{1}^{c}+$ $E P_{2}^{c}=v_{2}$. However, in many cases the contest is not symmetric. Hillman and Riley (1989) point out that an incumbent may value the prize more than a challenger. Nti (1999) lists a plethora of other examples. As $v_{1}$ increases beyond $v_{2}$ :

A. Neither contestant becomes more aggressive; contestant 1's expected expenditures are unchanged, while contestant 2's expected expenditures decline.

B. As a corollary, total expenditures are strictly decreasing in $v_{1}$, for $v_{1} \geq v_{2}$.

\footnotetext{
${ }^{1}$ For instance, in Konrad's (2009) textbook, incomplete information is discussed only sparingly. Likewise, in their collection of papers on rent seeking, Congleton, Hillman, and Konrad (2008) include only two papers, Wärneryd (2003) and Malueg and Yates (2004), which focus explicitly on incomplete information. Malueg and Yates (2004) concentrate on the Tullock contest; see Fey (2008) for a similar model. Wärneryd (2003) considers common value contests in which one contestant is uncertain of what that value is. Hurley and Shogren (1998) also examine one-sided incomplete information in a Tullock contest. Hillman and Riley (1989) briefly consider two-sided incomplete information in a deterministic contest, assuming contestants are symmetric ex ante. The current paper extends Hillman and Riley (1989) by allowing contestants to be asymmetric ex ante.
} 
The conclusion is that asymmetries among contestants are detrimental to competition, and, as a result, there is less dissipation of rent in the fight for the prize. ${ }^{2}$ Hillman and Riley (1989) thus conclude that asymmetries may explain the apparent under-dissipation of rent in real-world contests. As will be explained in Section 4, these comparative statics also provide a key building block in other studies, most prominently in Baye, Kovenock, and de Vries' (1993) seminal paper on the "exclusion principle". The robustness of the results is therefore of interest. ${ }^{3}$

The preceding contest is extreme on at least two dimensions. First, the contest is deterministic; there is no room for luck or random factors to influence the outcome. However, the comparative statics have recently been shown to be robust to changes in the contest success function. Che and Gale (2000) and Alcalde and Dahm (2010) each consider a class of (mutually exclusive) contest success functions for which the deterministic contest is a limiting case. In both cases, there is an equilibrium for which expenditures are given by (1) whenever the contest success function is "sufficiently close" to the deterministic success function, in the sense that a contestant's winning probability increases dramatically enough when he unilaterally increases his expenditures, starting from a position of equal expenditures. ${ }^{4}$

Second, the contest assumes information is complete. Although it may be reasonable to "suspect" that the incumbent values the prize more than the challenger, it is quite a stretch to claim that the challenger knows precisely how much the incumbent values the prize, and vice versa. It is also hard to imagine situations where the challenger is known to be the contestant with the lowest valuation with probability one.

In this paper, the robustness of the comparative statics to changes in the under-

\footnotetext{
${ }^{2} \mathrm{As}$ in much of the literature, the focus here is on the absolute level of rent dissipation, not the relative level. Moreover, this paper is concerned only with one-shot, simultaneous contests. See e.g. Amegashie (2006) and Konrad (2009) for discussions of dynamics in contests.

${ }^{3}$ This is not the first paper to question the robustness of these results. In reference to the complete information model, Anderson et al (1990) state that asymmetries "can lead to unintuitive comparative statics in a Nash equilibrium." They then develop a model with bounded rationality which exhibits very different comparative statics. For instance, a contestant who becomes stronger will respond by becoming more aggressive, in equilibrium. In contrast, contestants are rational in the current paper.

${ }^{4}$ Che and Gale (2000) consider difference-form contest success functions (CSFs), where the difference between expenditures determines winning probabilities. Alcalde and Dahm's (2010) class of CSFs include the Tullock CSF, where the probability of winning is determined by the ratio of expenditures. That is, if contestant $i$ 's expenditure is $e_{i}, i=1,2$, the probability that contestant 1 wins is $e_{1}^{r} /\left(e_{1}^{r}+e_{2}^{r}\right)$ or $\left(1+\left(\frac{e_{2}}{e_{1}}\right)^{r}\right)^{-1}$, where $r>0$. Remarkably, Alcalde and Dahm (2010) show that whenever $r \geq 2$, there is an equilibrium with the properties in (1). The all-pay auction corresponds to $r=\infty$. Nti (1999) obtains different results for $r \in(0,2)$; see also Baik (1994).
} 
lying information structure will be examined. The first contribution is to present a very general but surprisingly tractable incomplete information model, first introduced by Kirkegaard (2010a). Besides its tractability, a distinct advantage of the model is that the "strength" of a given contestant can be captured by a single variable, as $v_{i}$ does under complete information. It then becomes meaningful to speak of a "marginal increase" in the strength of a given contestant. ${ }^{5}$ Hence, comparative statics questions parallel to those considered before can be addressed. The model is presented in Section 2, while the comparative statics are described in Section 3.

It will be assumed that contestant $i$ draws a privately known valuation from a nondegenerate distribution with no mass-points and full support on $\left[0, \bar{v}_{i}\right], \bar{v}_{1} \geq \bar{v}_{2}>0$. The two distributions will be assumed to have similar shape. However, they are scaled differently, to fit onto their respective supports. It will be argued that $\bar{v}_{i}$ perfectly describes contestant $i$ 's strength in this particular model. For instance, the two distributions are identical if $\bar{v}_{1}=\bar{v}_{2}{ }^{6}$ Starting from $\bar{v}_{1}=\bar{v}_{2}$, the consequences of a marginal increase in $\bar{v}_{1}$ are:

a. The expected expenditures of both contestants strictly increase. In fact, contestant 1 's expected expenditures is strictly increasing in $\bar{v}_{1}$ globally.

b. As a corollary, total expected expenditures is strictly increasing in $\bar{v}_{1}$, when $\bar{v}_{1}$ is close to $\bar{v}_{2}$ and $\bar{v}_{1} \geq \bar{v}_{2}$. Total expenditures increase by exactly half as much as would be the case if both $\bar{v}_{1}$ and $\bar{v}_{2}$ increase by the same marginal amount.

Clearly, incomplete information gives rise to precisely the opposite comparative statics than complete information, at least when the asymmetry is small. Complete

\footnotetext{
${ }^{5}$ The difficulty with incomplete information in general is that a contestant is described by a distribution function, not by a scalar. So, a marginal change in strength translates into a "marginal change of a function", which is clearly ambiguous. However, with the model examined here, strength is captured by a single variable. At the same time, the model is quite general. Kirkegaard (2008) studies comparative statics in even more general (less structured) incomplete information all-pay auctions. The generality of that model, however, makes it more difficult to obtain results and to compare the available results to complete information contests. Nevertheless, Kirkegaard (2008) appears the first to provide an example with some of the features exhibited by the model examined here. The robustness of the results and their relationship to the literature on auctions with small asymmetries are discussed in Section 4.

${ }^{6}$ Although Hillman and Riley (1989) consider incomplete information, they assume contestants are symmetric ex ante - but not ex post - since valuations are drawn from the same distribution. The distinction between ex ante and ex post is moot in a complete information model. Thus, the appropriate generalization of the asymmetric complete information model is to an incomplete information model in which the asymmetries exist ex ante and ex post, or $\bar{v}_{1}>\bar{v}_{2}$. Such a model makes it possible to analyze what happens as one, and only one, contestant becomes stronger.
} 
information corresponds to the case where the distributions are degenerate at $\bar{v}_{i}$. It might be expected that (1) would obtain if almost all mass is concentrated close to $\bar{v}_{i}$. However, this is not the case - the comparative statics uncovered here apply as long as the distribution has positive density everywhere, regardless of how little, and no mass points. Thus, there is an intriguing "discontinuity" with respect to the information structure. Complete information is a knife-edge.

To illustrate, consider the distribution functions

$$
F_{i}(v)=\varepsilon \frac{v}{\bar{v}_{i}}+(1-\varepsilon)\left(\frac{v}{\bar{v}_{i}}\right)^{\frac{1}{\varepsilon}}, v \in\left[0, \bar{v}_{i}\right]
$$

where $\varepsilon$ is a small but positive number, $\varepsilon \in(0,1]$. The density is strictly positive everywhere, as long as $\varepsilon>0$. As $\varepsilon \rightarrow 0, F(v) \rightarrow 0$ for all $v \in\left[0, \bar{v}_{i}\right)$; hence, all mass is concentrated close to $v=\bar{v}_{i}$. If $\varepsilon=0$, the distribution is degenerate (information is complete). The point is that the comparative statics for $\varepsilon=0$ are very different from those arising for any strictly positive $\varepsilon$.

The complete information model is analytically simple and offers what appears to be a clear intuitive explanation for the comparative statics: asymmetries reduce competition because the weaker bidder is deterred by the stronger bidder. However, since the comparative statics are not robust, the intuition is evidently not robust either. The relationship between the complete and incomplete information models are discussed at length in Section 4, where the intuition for the new comparative statics are developed. ${ }^{7}$ Implications of the results are also discussed in Section 4, as is the connection to recent developments in the theory of auctions with small asymmetries.

\section{Model}

The contest is assumed to take the form of an all-pay auction. Hence, the auction terminology will be used. Two risk neutral bidders (contestants) are vying for a prize by submitting bids (committing effort to the contest). The highest bid wins, but both bidders pay their respective bids. A bidder is characterized by his type, which measures the monetary value he places on winning the prize. If a bidder with type $v$ bids $b$, his payoff is $v-b$ if he wins and $-b$ if he loses.

\footnotetext{
${ }^{7}$ The current paper is part of a more general research program on the consequences of incomplete information in contests. Kirkegaard (2010a) establishes that if a contest designer benefits from contestants' expenditures, then he may profit by handicapping the weak contestant. Kirkegaard (2010b) shows that preferential treatment may hurt a subset of the contestants it was supposed to help. Both results are impossible in complete information all-pay auctions.
} 
Bidder $i$ 's type is assumed to be drawn from a twice continuously differentiable distribution function $F_{i}$ with support $\left[0, \bar{v}_{i}\right], i=1,2$. The distribution function is assumed to have no mass points and to have strictly positive density, denoted by $f_{i}$, everywhere. The assumption that the lower end-point of the support is zero is deliberate and is of some importance. It is discussed in detail in Section 4.

Amann and Leininger (1996) provide an implicit characterization of equilibrium in this general model. Both bidders submit strictly positive bids with probability one (i.e. whenever their type is non-zero) and they both employ pure strategies that are strictly increasing in type. The equilibrium is unique.

However, more structure will be imposed on $F_{1}$ and $F_{2}$ in the current paper, in order to facilitate comparison with the complete information model. This model was first introduced by Kirkegaard (2010a). Assume that

$$
F_{i}(v)=F\left(\frac{v}{\bar{v}_{i}}\right), v \in\left[0, \bar{v}_{i}\right]
$$

where $F$ is a distribution function with the aforementioned properties on its support, $[0,1]$. Let $f>0$ denote its density. For future reference, let

$$
v_{i}^{s}=\frac{v}{\bar{v}_{i}}
$$

denote bidder $i$ 's "scale-adjusted" type. By (2), the two bidders' scale-adjusted types have the same distribution. Without loss of generality, assume $\bar{v}_{1} \geq \bar{v}_{2}$. The bidders are symmetric if and only if $\bar{v}_{1}=\bar{v}_{2}$. The distribution $F_{2}$ is a "scaled-down" version of $F_{1}$ if $\bar{v}_{1}>\bar{v}_{2}$; the two distributions have the exact same shape, but are of different scale to fit onto their respective supports. This can also be seen from the relationship

$$
F_{2}(v)=F_{1}\left(\frac{\bar{v}_{1}}{\bar{v}_{2}} v\right), v \in\left[0, \bar{v}_{2}\right]
$$

Note that $F_{1}$ first order stochastically dominates $F_{2}$ whenever $\bar{v}_{1}>\bar{v}_{2}$. That is, bidder 1 is less likely to have a low type than bidder 2 ; he is perceived to be stronger.

Let $E V_{i}$ denote the expected value of $v_{i}, i=1,2$, and let $E V_{F}$ denote the expected value of a draw from the distribution function $F$. Then, substituting the scaleadjusted type for the actual type (at the second-to-last equality) yields

$$
E V_{i}=\int_{0}^{\bar{v}_{i}} v f_{i}(v) d v=\int_{0}^{\bar{v}_{i}} \frac{v}{\bar{v}_{i}} f\left(\frac{v}{\bar{v}_{i}}\right) d v=\bar{v}_{i} \int_{0}^{1} v^{s} f\left(v^{s}\right) d v^{s}=\bar{v}_{i} E V_{F} .
$$


Thus, for a fixed distribution, $F, \bar{v}_{i}$ is a natural measure of the (absolute) strength of bidder $i$, as evidenced by (2) and (3). Therefore, $\bar{v}_{i}$ will be used as the basis for comparative statics. Let

$$
r_{i} \equiv \frac{\bar{v}_{j}}{\bar{v}_{i}}
$$

denote bidder $i$ 's relative strength, $i=1,2, i \neq j$. The lower $r_{i}$ is, the stronger bidder $i$ is compared to bidder $j$.

Compared to the benchmark model with complete information, the partial overlap of the supports implies that bidder 2 may happen to have a higher type than bidder 1 , even if $\bar{v}_{1}>\bar{v}_{2}$. However, the probability of this event can be made arbitrarily small by concentrating most of the probability mass of $F$ close to 1 (e.g. let $F$ be "very convex"). See also the example towards the end of the Introduction.

The remainder of this section is devoted to describing the equilibrium and the expected payments, for a fixed $\left(\bar{v}_{1}, \bar{v}_{2}\right)$ pair. A few preliminary results are also presented. Following this part, the comparative statics are almost trivial to derive. The comparative statics are described in a relatively succinct manner in Section 3. Detailed discussions are postponed until Section 4, where the important differences between the complete and incomplete information models are highlighted in an effort to bridge the gap between contest theory (with complete information) and auction theory (with incomplete information).

\subsection{Equilibrium and expected payments}

Since strategies are strictly increasing, bidder $i$ with type $v$ must submit the exact same bid as bidder $j$ with some, possibly different, type, $k_{i j}(v), i, j=1,2, i \neq j$. Thus, bidder $i$ wins if bidder $j$ 's type is below $k_{i j}(v)$. Since strictly positive bids are submitted whenever $v>0$, it must be the case that $k_{i j}(0)=0$. Likewise, $k_{i j}\left(\bar{v}_{i}\right)=\bar{v}_{j}$ must also hold. Otherwise, one bidder would irrationally be submitting bids that are strictly higher than is required to win with certainty.

Amann and Leininger's (1996) contribution is to supply an implicit characterization of the function $k_{i j}(v)$ for all $v \in\left[0, \bar{v}_{i}\right]$. The function is derived from the bidders' first order conditions, which form an autonomous system of differential equations. The boundary condition is $k_{i j}\left(\bar{v}_{i}\right)=\bar{v}_{j}$. In the present model, however, it is convenient to express the allocation in terms of the scale-adjusted types. Thus, define $k_{i j}^{s}\left(v^{s}\right)$ as the scale-adjusted type of bidder $j$ that bidder $i$ with scale-adjusted type $v^{s}$ would tie with, $v^{s} \in[0,1]$. Substituting the scale-adjusted type, as in (3), into 
Amann and Leininger's (1996) formula yields the relationship

$$
\int_{k_{i j}^{s}\left(v^{s}\right)}^{1} \frac{f(x)}{x} d x=r_{i} \int_{v^{s}}^{1} \frac{f(x)}{x} d x
$$

for all $v^{s} \in(0,1]$. The details are omitted from the present paper, but they can be found in Kirkegaard (2010a, Section 5).

For a fixed distribution, $F, k_{i j}^{s}\left(v^{s}\right)$ is determined exclusively by the relative strength of bidder $i, r_{i}$. As the purpose of the analysis is to vary $\bar{v}_{i}$, the function will be written as $k_{i j}^{s}\left(v^{s} \mid r_{i}\right)$ whenever the reliance on the relative strength needs to be made explicit. As bidder $i$ becomes stronger, or $r_{i}$ falls, $k_{i j}^{s}\left(v^{s}\right)$ must strictly increase to maintain the equality in $(4)$, for any $v^{s} \in(0,1)$. Thus, for a fixed scale-adjusted type, bidder $i$ wins more often the stronger he is.

If $\bar{v}_{1}>\bar{v}_{2}$ or $r_{i}<1, k_{12}^{s}\left(v^{s}\right)>v^{s}$ for all $v^{s} \in(0,1)$. In words, bidder 1 outbids a higher scale-adjusted type. Consequently, bidder 1's distribution of bids first order stochastically dominates that of bidder 2 ; bidder 1 is less likely to submit low bids. The same property holds in complete information all-pay auctions. As $\bar{v}_{1} \rightarrow \infty$, (4) implies that $k_{12}^{s}\left(v^{s}\right) \rightarrow 1$ for all $v^{s} \in(0,1)$. In the limit, bidder 1 wins with probability one. This is also the case in the complete information all-pay auction. Lemma 1 summarizes these preliminary results.

Lemma $1 k_{i j}^{s}\left(v^{s} \mid r_{i}\right)$ is strictly decreasing in $r_{i}$ for all $v^{s} \in(0,1)$. As $r_{i} \rightarrow 0$, $k_{i j}^{s}\left(v^{s} \mid r_{i}\right) \rightarrow 1$ for all $v^{s} \in(0,1)$, and as $r_{i} \rightarrow \infty, k_{i j}^{s}\left(v^{s} \mid r_{i}\right) \rightarrow 0$ for all $v^{s} \in(0,1)$.

Next, consider the expected bid or expected payment of bidder $i, E P_{i}\left(\bar{v}_{i}, \bar{v}_{j}\right)$. There are at least two ways of obtaining $E P_{i}\left(\bar{v}_{i}, \bar{v}_{j}\right)$. First, Amann and Leininger (1996) use $k_{i j}$ to derive bids as a function of types. The expectation of these bids can then be calculated. The alternative is to use Myerson's (1981) insight that the allocation in any given mechanism permits $E P_{i}\left(\bar{v}_{i}, \bar{v}_{j}\right)$ to be calculated directly, without first having to deduce bidding strategies. Kirkegaard (2010a) uses the second method to obtain the key property that $E P_{i}\left(\bar{v}_{i}, \bar{v}_{j}\right)$ is separable in the bidder's absolute strength, $\bar{v}_{i}$, and relative strength, $r_{i}$. Lemma 2 summarizes Kirkegaard's (2010a) results.

Lemma 2 Bidder $i$ 's payment can be written

$$
E P_{i}\left(\bar{v}_{i}, \bar{v}_{j}\right)=\bar{v}_{i} \times E P^{s}\left(r_{i}\right)
$$

where

$$
E P^{s}\left(r_{i}\right)=r_{i} \int_{0}^{1} k^{s}\left(v^{s} \mid r_{i}\right)\left(1-F\left(v^{s}\right)\right) f\left(v^{s}\right) d v^{s} .
$$

$E P^{s}$ is differentiable in $r_{i}$ and has the property that $E P^{s \prime}(1)>0$. 
Proof. Here it will be proven only that $E P_{i}\left(\bar{v}_{i}, \bar{v}_{j}\right)$ is separable, as stated in (5). Proving the remainder of the Lemma is more involved. The proofs are omitted from this paper, but can be found in Lemma 3 in Kirkegaard (2010a).

Since a bidder with type zero earns zero rent, it follows from Myerson (1981) that bidder $i$ 's ex ante expected payment is

$$
\begin{aligned}
E P_{i}\left(\bar{v}_{i}, \bar{v}_{j}\right) & =\int_{0}^{\bar{v}_{i}}\left(v-\frac{1-F_{i}(v)}{f_{i}(v)}\right) F_{j}\left(k_{i j}\left(v \mid r_{i}\right)\right) f_{i}(v) d v \\
& =\bar{v}_{i} \int_{0}^{\bar{v}_{i}}\left(\frac{v}{\bar{v}_{i}}-\frac{1-F\left(\frac{v}{\bar{v}_{i}}\right)}{f\left(\frac{v}{\bar{v}_{i}}\right)}\right) F\left(\frac{k_{i j}\left(v \mid r_{i}\right)}{\bar{v}_{j}}\right) \frac{1}{\bar{v}_{i}} f\left(\frac{v}{\bar{v}_{i}}\right) d v .
\end{aligned}
$$

Substituting in the scale-adjusted type, $v^{s}=\frac{v}{\bar{v}_{i}}$, then yields

$$
E P_{i}\left(\bar{v}_{i}, \bar{v}_{j}\right)=\bar{v}_{i} \int_{0}^{1}\left(v^{s}-\frac{1-F\left(v^{s}\right)}{f\left(v^{s}\right)}\right) F\left(k_{i j}^{s}\left(v^{s} \mid r_{i}\right)\right) f\left(v^{s}\right) d v,
$$

which takes the form (7).

Before examining the implications of Lemma 2, it is interesting to compare (5) and its counterpart in the complete information model, (1). The latter can also be written in a separable form,

$$
E P_{i}^{c}=v_{i} \times E P^{c}\left(r_{i}^{c}\right)
$$

where $r_{i}^{c} \equiv v_{j} / v_{i}$, and

$$
E P^{c}\left(r_{i}^{c}\right)= \begin{cases}\frac{1}{2} r_{i}^{c} & \text { if } r_{i}^{c} \leq 1 \\ \frac{1}{2}\left(r_{i}^{c}\right)^{-1} & \text { otherwise }\end{cases}
$$

is a continuous function. However, $E P^{c}$ is not differentiable at $r_{i}^{c}=1$, where it peaks. In contrast, $E P^{s}$ does not peak at $r_{i}=1$. This difference helps explain the different results in the two models (see Section 4 ).

Inserting (6) into (5) yields

$$
E P_{i}\left(\bar{v}_{i}, \bar{v}_{j}\right)=\bar{v}_{j} \int_{0}^{1} k^{s}\left(v^{s} \mid r_{i}\right)\left(1-F\left(v^{s}\right)\right) f\left(v^{s}\right) d v^{s} .
$$

It is sometimes emphasized that all rents are dissipated in a symmetric complete information all-pay auction, but not in a symmetric incomplete information all-pay 
auction. Bidders earn "information rent" in the latter. Nevertheless, from (9) observe that if $\bar{v}_{i}=\bar{v}_{j}$,

$$
E P_{i}\left(\bar{v}_{i}, \bar{v}_{i}\right)=\frac{1}{2} \times \bar{v}_{i} \int_{0}^{1} 2 v^{s}\left(1-F\left(v^{s}\right)\right) f\left(v^{s}\right) d v^{s}
$$

Hence, as in the complete information contest, each bidder, in expectation, pays half the expected value of the second highest type. The difference is that there is no wedge between the highest and second highest type in a symmetric complete information model.

There are also some interesting similarities between the two models at the other extreme, when the asymmetry between bidders is large. In the complete information model, from (1),

$$
E P_{1}^{c} \rightarrow \frac{v_{2}}{2} \text { and } E P_{2}^{c} \rightarrow 0 \text { as } v_{1} \rightarrow \infty
$$

Turning to the incomplete information model, Lemma 1 describes the behavior of $k_{i j}^{s}$ in the limit. Then, (9) implies

$$
E P_{1} \rightarrow \bar{v}_{2} \frac{1}{2} \times \int_{0}^{1} 2\left(1-F\left(v^{s}\right)\right) f\left(v^{s}\right) d v^{s}=\frac{\bar{v}_{2}}{2} \text { as } \bar{v}_{1} \rightarrow \infty
$$

since the term under the integration is a density (the density of the lowest scaleadjusted type), which integrates to one. Likewise, since $k_{21}^{s}\left(v^{s}\right) \rightarrow 0$ as $\bar{v}_{1} \rightarrow \infty$ (bidder 2 never wins), it must also be the case that

$$
E P_{2} \rightarrow 0 \text { as } \bar{v}_{1} \rightarrow \infty .^{8}
$$

Thus, the two models have similar features at the "corners" (no asymmetry or extreme asymmetry). However, they differ markedly in their behavior as one moves from one corner to the other. The comparative statics are described next.

\section{Comparative Statics}

Consider the individual responses to changes in $\bar{v}_{i}$.

\footnotetext{
${ }^{8} \mathrm{~A}$ formal proof of this follows from Myerson (1981). It is immediate from his formulation of the expected payment that it must converge to zero as the winning probability converges to zero for almost all types (recall that $k_{21}^{s}\left(\bar{v}_{2}\right)=\bar{v}_{1}$, so bidder 2 with type $\bar{v}_{2}$ wins with probability one; all other types wins with probability converging to zero). It is intuitive that bidder 2 would not be willing to pay anything if he has no chance of winning.
} 
Proposition $1 E P_{i}\left(\bar{v}_{i}, \bar{v}_{j}\right)$ is strictly increasing in $\bar{v}_{i}$ and non-monotonic in $\bar{v}_{j}$, with

$$
\frac{\partial E P_{i}\left(\bar{v}_{i}, \bar{v}_{j}\right)}{\partial \bar{v}_{j}}>0 .
$$

Proof. By Lemma $1, k_{i j}^{s}\left(v^{s}\right)$ is strictly increasing in $\bar{v}_{i}$, for all $v^{s} \in(0,1)$. Equation (9) then implies that $E P_{i}\left(\bar{v}_{i}, \bar{v}_{j}\right)$ must be strictly increasing in $\bar{v}_{i}$ as well. From (5),

$$
\frac{\partial E P_{i}\left(\bar{v}_{i}, \bar{v}_{j}\right)}{\partial \bar{v}_{j}}=\bar{v}_{i} \times E P^{s \prime}\left(r_{i}\right) \frac{1}{\bar{v}_{i}}=E P^{s \prime}\left(r_{i}\right) .
$$

By Lemma 2, this derivative is strictly positive when evaluated at $\bar{v}_{j}=\bar{v}_{i}$. At $\bar{v}_{j}=\bar{v}_{i}, E P_{i}$ is strictly increasing in $\bar{v}_{j}$ and strictly positive. As explained in Section 2 , however, it converges to zero as $\bar{v}_{j} \rightarrow \infty$. Hence, it cannot be monotonic in $\bar{v}_{j}$.

In a complete information all-pay auction, $E P_{i}^{c}$ is increasing in $v_{i}$ as long as $v_{i}<v_{j}$. Thereafter, $E P_{i}^{c}$ is unchanged if $v_{i}$ increases further. In contrast, in an incomplete information contest, $E P_{i}$ is globally strictly increasing in $\bar{v}_{i} . E P_{i}^{c}$ is nonmonotonic in $v_{j}$; increasing in $v_{j}$ as long as $v_{j}<v_{i}$, and decreasing thereafter. While $E P_{i}$ is also non-monotonic in $\bar{v}_{j}$ in an incomplete information contest, it does not attain its maximum at $\bar{v}_{j}=\bar{v}_{i}$. In particular, starting from a point of symmetry, $\bar{v}_{j}=\bar{v}_{i}$, bidder $i$ actually becomes more aggressive (in expectation) when his rival becomes marginally stronger, which is precisely the opposite of what happens in the complete information all-pay auction. Figure 1 illustrates the difference between the two models. A discussion of the intuition is postponed until Section 4.

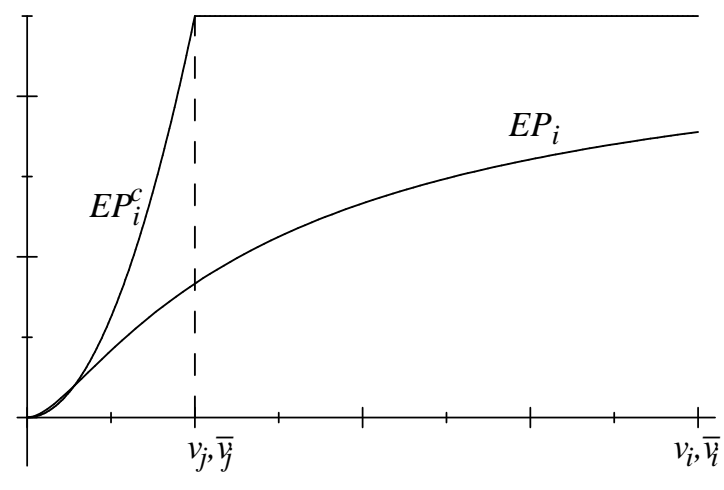

(a) $E P_{i}^{c}, E P_{i}$ as functions of $v_{i}, \bar{v}_{i}$.

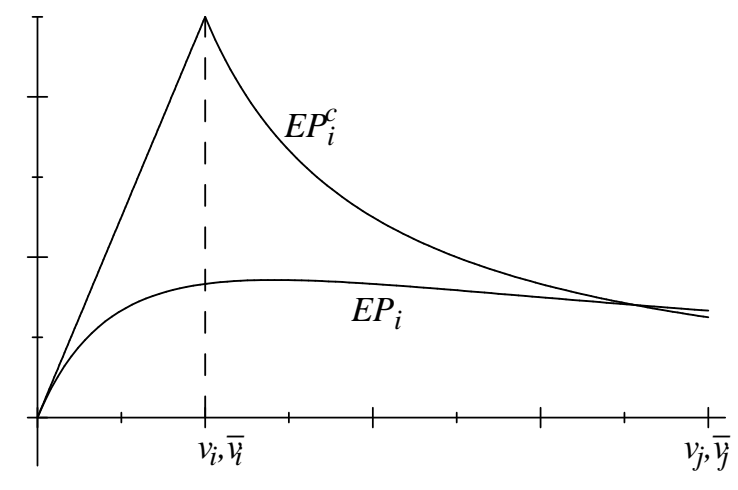

(b) $E P_{i}^{c}, E P_{i}$ as functions of $v_{j}, \bar{v}_{j}$.

Figure 1: Comparative statics in complete and incomplete information models. ${ }^{9}$

${ }^{9}$ Formally, it has not been proven that $E P_{i}$ in general is single-peaked in $\bar{v}_{j}$. Figure 1 depicts actual expected payments when $F$ is a uniform distribution, in which case $E P_{i}$ is in fact singlepeaked in $\bar{v}_{j}$. Recall that $E P_{i}$ and $E P_{i}^{c}$ converge to each other as $\bar{v}_{i} \rightarrow \infty$ or $\bar{v}_{j} \rightarrow \infty$. 
Since both bidders becomes more aggressive as one bidder becomes stronger, starting from symmetry, it is an obvious corollary that total payments must increase as well. Contrary to the complete information model, rent dissipation may be an increasing problem as bidders become asymmetric. In the present model, the increase in total spending can in fact be quantified.

Proposition 2 Assume that $\bar{v}_{1}=\bar{v}_{2}$. Then, a marginal increase in $\bar{v}_{1}$ leads total expected payments to increase by exactly half as much as would be the case if both $\bar{v}_{1}$ and $\bar{v}_{2}$ were to increase by the same marginal amount.

Proof. Total expected payments in the auction are

$$
E T\left(\bar{v}_{1}, \bar{v}_{2}\right)=\bar{v}_{1} \times E P^{s}\left(r_{1}\right)+\bar{v}_{2} \times E P^{s}\left(r_{2}\right),
$$

implying that

$$
\frac{\partial E T\left(\bar{v}_{1}, \bar{v}_{2}\right)}{\partial \bar{v}_{1}}=E P^{s}\left(r_{1}\right)+\bar{v}_{1} \times E P^{s \prime}\left(r_{1}\right) \frac{-\bar{v}_{2}}{\left(\bar{v}_{1}\right)^{2}}+\bar{v}_{2} \times E P^{s \prime}\left(r_{2}\right) \frac{1}{\bar{v}_{2}} .
$$

Evaluated at $\bar{v}_{1}=\bar{v}_{2}$ or $r_{1}=r_{2}=1$, the derivative is

$$
\frac{\partial E T\left(\bar{v}_{1}, \bar{v}_{1}\right)}{\partial \bar{v}_{1}}=E P^{s}(1)-E P^{s \prime}(1)+E P^{s \prime}(1)=E P^{s}(1)
$$

In comparison, if $\bar{v}_{1}=\bar{v}_{2}=\bar{v}$, the total expected payments are $2 \bar{v} E P^{s}(1)$, the derivative of which is $2 E P^{s}(1)$ with respect to $\bar{v}$. This completes the proof.

Proposition 2 describes the consequences of small asymmetries. Given the findings in Section 2.1, the expected total payments can be quantified when there is no asymmetry, or when the asymmetry is extreme.

Proposition 3 If bidders are symmetric, or $\bar{v}_{1}=\bar{v}_{2}$, total expected payments equals the expected value of the second highest type,

$$
\operatorname{ET}\left(\bar{v}_{2}, \bar{v}_{2}\right)=\bar{v}_{2} \int_{0}^{1} 2 v^{s}\left(1-F\left(v^{s}\right)\right) f\left(v^{s}\right) d v^{s} .
$$

As bidder 1 becomes stronger, total expected payments converge to $\bar{v}_{2} / 2$,

$$
E T\left(\bar{v}_{1}, \bar{v}_{2}\right) \rightarrow \frac{\bar{v}_{2}}{2} \text { as } \bar{v}_{1} \rightarrow \infty .
$$


Proof. The first part follows from (10), the second from (11) and (12).

The relative size of (14) and (15) depends on the underlying distribution, $F$. For instance, if $F$ is the uniform distribution on $[0,1], E T\left(\bar{v}_{2}, \bar{v}_{2}\right)=\bar{v}_{2} / 3$. In this case, an extremely asymmetric all-pay auction yields higher higher total payments than a symmetric all-pay auction. This conclusion holds whenever $F$ is stochastically dominated by the uniform distribution, as when $F$ is concave. ${ }^{10}$

On the other hand, if $F$ has most of its mass close to $v^{s}=1, \operatorname{ET}\left(\bar{v}_{2}, \bar{v}_{2}\right)$ will be close to $\bar{v}_{2}$. In this case, a symmetric auction yields higher expected payments than an extremely asymmetric one. In conclusion, large asymmetries may cause more or less rent to be dissipated, compared to a symmetric contest. However, small asymmetries will unambiguously cause more rent to be dissipated, by Proposition 2 .

The distribution function $F$ has most of its mass close to $v^{s}=1$ when information is "almost complete", in which case rent dissipation will be almost complete as well, in a symmetric contest. Thus, this feature along with the ones mentioned in Section 2.1 are continuous in the information structure. The comparative statics are not.

\section{Discussion and conclusions}

The paper concludes with a discussion of some implications of the results. The complete and incomplete information environments are then compared in terms of the strategic considerations inherent in each. The differences between the properties of the all-pay auction as a mechanism in the two models are highlighted. In the process, intuitive explanations of the comparative statics in the two models are developed and the robustness of the results to other ways of modelling incomplete information is discussed. Another comparative statics is also presented.

\subsection{The value of (a)symmetric competition}

Baye, Kovenock, and de Vries (1993) consider a contest in which a contest designer benefits from bidders' expenditures. Their model assumes complete information, but allows for $n$ bidders. As before, let $v_{i}$ denote bidder $i$ 's valuation. For the purpose of exposition, assume that $v_{1}>v_{2}>\ldots>v_{n}$, in which case the equilibrium is unique.

\footnotetext{
${ }^{10}$ Once again, it has not been proven that $\operatorname{ET}\left(\bar{v}_{1}, \bar{v}_{2}\right)$ is single-peaked in $\bar{v}_{1}$, although it is when $F$ is the uniform distribution. Whenever ET is single peaked in $\bar{v}_{1}$ and $E T\left(\bar{v}_{2}, \bar{v}_{2}\right) \leq \bar{v}_{2} / 2$, it must be the case that a symmetric auction produces lower payments than any asymmetric auction. When $F$ is uniform and $\bar{v}_{2}=1, E T\left(\bar{v}_{1}, 1\right)$ attains its maximum at $\bar{v}_{1} \approx 12.3$. In other words, bidder 1 must be at least 12 times as strong as bidder 2 in order for further increases in $\bar{v}_{1}$ to reduce total payments. This conclusion is in sharp contrast to the one obtained under complete information.
} 
Baye, Kovenock, and de Vries (1993) famously show that the contest designer may benefit from excluding a subset of bidders from the contest, specifically the stronger ones.

There are two key steps in Baye, Kovenock, and de Vries' (1993) proof. First, in an all-pay auction with $v_{1}>v_{2}>\ldots>v_{n}$, only bidder 1 and bidder 2 will be active. All other bidders will be scared off, and will not participate (so there is no need to explicitly exclude these bidders). Second, in the complete information all-pay auction, revenue is decreasing in the stronger bidder's valuation. Thus, by excluding bidder 1 , the contest designer may create a more even competition between bidder 2 and bidder 3 . Of course, he may continue to exclude bidder 2 , to trigger a competition between bidders 3 and 4 , and so on.

Now imagine information is incomplete. It is known that the first step in the proof does not hold in an incomplete information contest; see Parreiras and Rubinchik (2010) and Kirkegaard (2010b). In the current paper, it has been shown that the second step does not hold either. Naturally, this does not imply that Baye, Kovenock, and de Vries' (1993) exclusion principle is not robust, only that the method of proof does not carry over. A new proof - or a counterexample - is not pursued in this paper. However, these observations coupled with the results of this paper hopefully demonstrate that the theory of contests with incomplete information constitutes a rich research topic.

The results in this paper do, however, have direct implications for the selection of bidders into a contest. Imagine a situation where a designer has to select a pair of finalists. For some reason (e.g. affirmative action or personal preference) he is obliged to admit bidder 3 as one of the finalists. In a complete information contest with $n=3$ and $v_{1}>v_{2}>v_{3}$, a contest designer who benefits from bidders' payments is better off selecting bidder 2 as the remaining finalist, to create a more even contest. However, this is not necessarily the case if information is incomplete; it may be more profitable to create an uneven contest.

\subsection{Strategic effects}

The strategic effects of increased asymmetry are different in the complete and incomplete information models.

In the complete information model, bidders are willing to use mixed strategies because they are made indifferent between all bids in $\left[0, v_{2}\right]$. If bidder 2 does not change his strategy when bidder 1 becomes stronger, bidder 1 would find it optimal to bid $v_{2}$ with probability one. To restore the equilibrium, bidder 2 has to submit low bids more often. In equilibrium, bidder 1's strategy is unchanged, because the initial 
strategy already makes bidder 2 willing to randomize. The result is unambiguously a decrease in expected total expenditures.

As discussed in Amann and Leininger (1996), incomplete information purifies the strategies. That is, each bidder uses a pure strategy (which is a function of his type). However, the differences run deeper than that. For instance, bidders earn information rent, from which it is possible to conclude right away that the highest possible bid, $\bar{b}$, must be strictly lower than $\bar{v}_{2}$. Now, if bidder 2 does not change his strategy as $\bar{v}_{1}$ increases to $\bar{v}_{1}^{\prime}$, bidder 1 would find it optimal to bid precisely $\bar{b}$ if he happens to draw a type between $\bar{v}_{1}$ and $\bar{v}_{1}^{\prime}$. In other words, a mass of types would bid $\bar{b}$. Since $\bar{v}_{2}>\bar{b}$, bidder 2 would respond by bidding marginally above $\bar{b}$ if his type is close to $\bar{v}_{2}$. In equilibrium, this effect forces $\bar{b}$ to increase. As the bids submitted by high types change, lower types should also be expected to revise their bids.

Kirkegaard (2008) examines what happens as one bidder becomes stronger in the sense that he draws a type from a stochastically dominant distribution. Kirkegaard (2008) shows that this bidder's new bid distribution will stochastically dominate the old bid distribution. A bidder who becomes stronger is more likely to bid more aggressively, implying that his expected payment increases. This result is independent of how $F_{1}$ and $F_{2}$ are related. Thus, the first part of Proposition 1 is a special case.

However, it is harder to obtain results for the bidder who sees his opponent grow stronger. To understand why, note that the strategic considerations depend on the bidder's type. In particular, the trade-off between bidding higher and winning with a higher probability is determined by the bidder's type. Thus, a bidder would react differently to a rival's more aggressive behavior depending on whether his type is high or low. The previous argument shows that bidder 2 with a type close to $\bar{v}_{2}$ is forced to bid more aggressively when he sees bidder 1 grow stronger. However, a low type, one who does not value winning very much, may be better off bidding more cautiously when his rival becomes more aggressive.

Kirkegaard (2008) examines another one-variable model. His results confirms the intuition described above. Specifically, consider some strictly increasing function, $H(v)$, with $H(0)=0$. Assume that $F_{1}$ can be written

$$
F_{1}(v)=\frac{H(v)}{H\left(\bar{v}_{1}\right)}, v \in\left[0, \bar{v}_{1}\right],
$$

i.e., $F_{1}$ is a truncation of $H$. In this case, $\bar{v}_{1}$ is once again indicative of bidder 1 's strength. Assuming the support of $F_{2}$ is $\left[0, \bar{v}_{2}\right]$, as in the current paper, Kirkegaard (2008) shows that as $\bar{v}_{1}$ increases, bidder 2 will bid less aggressively if his type is low, but more aggressively if his type is high. The new bidding strategy (and the distribution of bids) will cross the old one exactly once. Unfortunately, in general, 
it is not possible to say whether bidder 2's expected payment increases or decreases, within that model. Thus, in general it is not possible to establish whether total payments increase or decrease either. However, the recent literature on auction with small asymmetries reveal that such inferences may be possible for "marginal changes" in bidder 1's distribution. This point is discussed more thoroughly in Section 4.5.

The advantages of the model presented in this paper is its close relationship to the complete information model, as described in Section 2, as well as its supreme tractability. In particular, the model allows the conclusion that bidder 2 becomes more aggressive, on balance (that it, in expectation), when his opponent grows stronger starting from a point of symmetry. Kirkegaard (2010a) uses the same model to examine the use of handicaps in all-pay auctions (see Section 4.4).

\subsection{All-pay auctions as mechanisms}

The complete and incomplete information models differ not only in terms of the strategic considerations of the bidders. Another difference between the models is in the effectiveness with which the all-pay auction robs bidders of rent. From a mechanism design perspective, a key determinant of expenditures in any auction or contest is the allocation. Nevertheless, in the complete information model, the allpay auction extracts less rent from bidders than other mechanisms with the exact same allocation. In particular, bidder 1 earns payoff of $v_{1}-v_{2}$, in expectation, when $v_{1} \geq v_{2}$. His "participation constraint" is not binding. It becomes "more slack" as $v_{1}$ grows, meaning that the effectiveness of the all-pay auction at extracting rent diminishes as bidder 1 grows stronger.

Bidder 1 would be willing to pay a fee of up to $v_{1}-v_{2}$ to participate in the auction, even though such a fee would not change bidding behavior or the allocation. With such a fee, the total expected payment would be

$$
E T_{f e e}^{c}=\left(v_{1}-v_{2}+\frac{v_{2}}{2}\right)+\frac{v_{2}^{2}}{2 v_{1}}
$$

which is clearly increasing in $v_{1}, v_{1} \geq v_{2}$. Thus, there are two different mechanisms all-pay auctions with or without fees - for which the bidding strategies are the same and for which strategies change in the same manner when one contestant grows stronger. Nevertheless, the payments and comparative statics are different.

In comparison, the participation constraints are binding in the incomplete information model. This is due to the deliberate assumption that the lowest type is zero. A bidder with type zero earns zero rent. Hence, no other mechanism could extract more rent from bidders without changing the allocation. Contrary to the complete information model, this property holds regardless of how strong the bidder is. 
With these observations, it is now possible to present an alternative intuitive explanation for the comparative statics in the two models.

\subsection{Absolute and relative strength: A perturbation}

When $\bar{v}_{1}$ changes, bidder 1's absolute strength and relative strength change as a consequences. Given (5) and (7), it is worthwhile to decompose the two effects.

To get at the role of the relative strength, consider first a symmetric incomplete information contest, with $\bar{v}_{1}=\bar{v}_{2}=1$. Now, perturb the model to permit bidder 1 an advantage. Specifically, bidder 1 wins the all-pay auction if his bid, $b_{1}$, exceeds $h_{1} b_{2}$, where $b_{2}$ is bidder 2's bid and $h_{1} \in(0,1]$ measures the size of bidder 2's handicap relative to bidder 1. As documented in Kirkegaard (2010a), it turns out that $h_{1}$ determines the scale-adjusted allocation in precisely the same way that $r_{1}$ does in the asymmetric model; $h_{1}$ takes the place of $r_{1}$ in (4). Thus, changes in handicaps will have the same effects as changing the relative strength of the bidders, but without changing the absolute strengths. Since $r_{2}=r_{1}^{-1}$ (bidder 1's advantage or relative strength is the reciprocal of bidder 2's handicap or relative strength), total expected payments in the perturbed model are given by

$$
E T_{p}^{s}\left(r_{1}\right)=E P^{s}\left(r_{1}\right)+E P^{s}\left(r_{1}^{-1}\right)
$$

Crucially, $E T_{p}^{s \prime}(1)=0$. From an auction perspective, this is unsurprising. Given mild regularity assumptions on $F$, the all-pay auction, being efficient ( $\operatorname{since} \bar{v}_{1}=\bar{v}_{2}$ ) and leaving no rent for the lowest types, produces the highest expected payments that are possible in any mechanism where the prize is "sold" with probability one. ${ }^{11}$ Any change to the rules of the games, such as imposing handicaps, cannot increase the total expected payments. Since $E T_{p}^{s^{\prime}}(1)=0$, small perturbations from symmetry are essentially irrelevant.

Recall that the total expected payments in the unperturbed model is

$$
E T\left(\bar{v}_{1}, \bar{v}_{2}\right)=\bar{v}_{1} E P^{s}\left(r_{1}\right)+\bar{v}_{2} E P^{s}\left(r_{1}^{-1}\right)=\left(\bar{v}_{1}-\bar{v}_{2}\right) E P^{s}\left(r_{1}\right)+\bar{v}_{2} E P_{p}^{s}\left(r_{1}\right)
$$

The significance of $E T_{p}^{s \prime}(1)=0$ is that a small change in the relative strength of the bidders, starting from symmetry, has no first order effect. Thus, when $\bar{v}_{1}$ increases marginally, starting from $\bar{v}_{1}=\bar{v}_{2}$, it is only the change in bidder 1's absolute strength

\footnotetext{
${ }^{11}$ This result is due to Myerson (1981). Note that Myerson's (1981) regularity condition has not been imposed here. Hence, $E T_{p}^{s \prime}(1)=0$ is a stronger result. Even if the regularity assumption is not satisfied, $E T_{p}^{s}$ has a stationary point at $r_{1}=1$.
} 
that is important. This explains why the impact of a marginal change in $\bar{v}_{1}$,

$$
\frac{\partial E T\left(\bar{v}_{1}, \bar{v}_{2}\right)}{\partial \bar{v}_{1} \mid \bar{v}_{1}=\bar{v}_{2}}=E P^{s}(1)
$$

is exactly half of what the impact would be if both $\bar{v}_{1}$ and $\bar{v}_{2}$ changed by the same marginal amount.

In comparison, consider the same thought experiment conducted on the complete information model, with $v_{1}=v_{2}=1$. Once again, a handicap effects the all-pay auction in the same manner as changes in the relative strength of the bidders does. This time, from (8) and $r_{2}^{c}=\left(r_{1}^{c}\right)^{-1}$,

$$
E T_{p}^{c}\left(r_{1}\right)=E P^{c}\left(r_{1}^{c}\right)+E P^{c}\left(\left(r_{1}^{c}\right)^{-1}\right)= \begin{cases}r_{1}^{c} & \text { if } r_{i}^{c} \leq 1 \\ \left(r_{1}^{c}\right)^{-1} & \text { otherwise }\end{cases}
$$

Clearly, $E T_{p}^{c}\left(r_{1}\right)$ is maximized at $r_{1}=1$, just as in the incomplete information model. The reason is once again that the all-pay auction maximizes expected payments among all mechanisms. However, $E T_{p}^{c}\left(r_{1}\right)$ has a kink at $r_{1}=1-$ a small perturbation does in fact have a first order effect. In this model, a perturbation changes the relative strength but it also allows bidder 1 to appropriate rent. As discussed in the previous subsection, bidder 1's participation constraint becomes slack. This opens a gap between the all-pay auction and the payment-maximizing mechanism with the same allocation. It is this effect that accounts for the kink. ${ }^{12}$ This feature is absent from the incomplete information model, where the participation constraint remains binding for the lowest types.

\subsection{Robustness to other models of incomplete information}

Fibich et al (2004) consider a general incomplete information model in which $F_{1}$ and $F_{2}$ have the same support, $[\underline{v}, \bar{v}]$. Given some "benchmark" distribution, $F$, on $[\underline{v}, \bar{v}]$, assume that $F_{i}(v)$ can be written $F_{i}(v)=F(v)+\varepsilon H_{i}(v)$, where $\varepsilon$ is a small number and $H_{i}(\underline{v})=H_{i}(\bar{v})=0$. The two bidders are symmetric if $\varepsilon=0$. Under certain conditions, Fibich et al (2004) show that a marginal increase in $\varepsilon$, starting from zero, has the same first-order effect on expected revenue regardless of the mechanism that is being used. One of the key conditions is that a bidder with type $\underline{v}$ earns zero rent. Thus, their results are applicable to the all-pay auction only if $\underline{v}=0$. Lebrun (2009) generalizes some of the results in Fibich et al (2004).

\footnotetext{
${ }^{12}$ In the complete information model with a fee described before, $E P_{\text {fee }}^{c}\left(r_{i}^{c}\right)=\left(1-.5 r_{i}^{c}\right)$ if $r_{i} \leq 1$ and $E P_{f e e}^{c}\left(r_{i}^{c}\right)=.5\left(r_{i}^{c}\right)^{-1}$ otherwise. Thus, $E P_{f e e}^{c}\left(r_{1}^{c}\right)+E P_{f e e}^{c}\left(\left(r_{1}^{c}\right)^{-1}\right)=1$, which is obviously differentiable and flat at $r_{1}=1$.
} 
To compare with the current paper, assume that $H_{1}(v) \leq 0$ and $H_{2}(v)=0$ for all $v \in[\underline{v}, \bar{v}]$. Then, an increase in $\varepsilon$ means that bidder 1 becomes stronger in the sense of first order stochastic dominance. It is straightforward to show that in this case revenue must increase in a second price auction. Thus, the result in Fibich et al (2004) and Lebrun (2009) imply that total expenditures must also increase in an all-pay auction. In other words, the result that asymmetries increase total rent dissipation is robust.

However, Fibich et al (2004) and Lebrun (2009) are concerned only with total expenditures. In the model presented here, it is possible to establish the stronger result that both bidders bid more aggressively when one becomes marginally stronger. ${ }^{13}$ Moreover, the supports are permitted to be different, thereby extending the scope of Fibich et al (2004) and Lebrun (2009). ${ }^{14}$ Arguably, the assumption of different supports, $\bar{v}_{1} \geq \bar{v}_{2}$, also makes the comparison to the complete information model with asymmetric valuations, $v_{1} \geq v_{2}$, more immediate. Finally, the current paper explores the intuitive differences between complete and incomplete information models.

It should be noted that as $\varepsilon$ or $\bar{v}_{1}$ increases in the incomplete information model, the expected value of the second highest type increases. This is not the case in the complete information model when $v_{1}$ increases, $v_{1} \geq v_{2} \cdot{ }^{15}$ Note that expected revenue in a second price auction is identical to the expected value of the second highest type, regardless of the information structure. The results mentioned above signify that total expenditures in the all-pay auction increases at exactly the same pace as the expected value of the second highest type when $\bar{v}_{1}$ increases marginally, starting from $\bar{v}_{1}=\bar{v}_{2}$. However, when $F$ is uniform it can be verified that total expected payments are strictly higher than the expected value of the second highest type whenever $\bar{v}_{1}>\bar{v}_{2} \cdot{ }^{16}$ Hence, the increase in the expected value of the second highest type does not account for the difference in the comparative statics between the complete and incomplete information models.

\footnotetext{
${ }^{13}$ In fact, individual expenditures may differ from mechanism to mechanism, even though their sum does not. For instance, in the uniform model, bidder 2 will pay less in a second price auction as $\bar{v}_{1}$ increases, for $\bar{v}_{1} \geq \bar{v}_{2}$. This is not the case in the all-pay auction (Proposition 1 ).

${ }^{14}$ Proposition 2 is consistent with Fibich et al (2004) and Lebrun (2009). It can easily be verified that expected revenue in a second price auction increases by exactly $E P^{s}(1)$ when $\bar{v}_{1}$ increases marginally above $\bar{v}_{2}$, just as in the all-pay auction.

${ }^{15}$ In the incomplete information model, however, the accepted notion of "getting stronger" is to draw a type from a stochastically dominant distribution, which must necessarily cause the expected value of the lowest type to increase (when the supports of $F_{1}$ and $F_{2}$ overlap).

${ }^{16}$ Thus, the all-pay auction yields higher expected payments than the second-price auction. In other words, more resources are expended in the contest than in any efficient mechanism. The exact opposite is the case in the complete information model, where the all-pay auction yields lower payments that an efficient mechanism.
} 


\subsection{Other comparative statics}

So far, only a limited number of comparative statics have been compared for the two models. The differences between the conclusions obtained are sufficient to demonstrate the lack of robustness of the complete information model. However, there are other comparative statics that could be derived to meaningfully shed light on the consequences of increased asymmetry. For instance, Konrad (2009) considers a complete information Tullock contest in which $v_{1}=v+d$ and $v_{2}=v-d, d \in[0, v]$. Here, changes in $d$ does not change the average valuation of the bidders.

In the context of the current paper, it is easy to see that total expenditures in a complete information all-pay auction is strictly decreasing in $d$. However, this is not the case in the incomplete information model.

Proposition 4 Consider the incomplete information model and assume that $\bar{v}_{1}=$ $\bar{v}+d, \bar{v}_{2}=\bar{v}-d, d \in[0, \bar{v}]$. Then, small asymmetries are irrelevant, or

$$
\left.\frac{\partial E T(\bar{v}+d, \bar{v}-d)}{\partial d}\right|_{\mid d=0}=0 .
$$

Proof. The Proposition follows directly from

$$
\begin{aligned}
& E T(\bar{v}+d, \bar{v}-d)=(\bar{v}+d) \times E P^{s}\left(\frac{\bar{v}-d}{\bar{v}+d}\right)+(\bar{v}-d) \times E P^{s}\left(\frac{\bar{v}+d}{\bar{v}-d}\right) \\
& \quad=\bar{v} \times\left(E P^{s}\left(\frac{\bar{v}-d}{\bar{v}+d}\right)+E P^{s}\left(\frac{\bar{v}+d}{\bar{v}-d}\right)\right)+d\left(E P^{s}\left(\frac{\bar{v}-d}{\bar{v}+d}\right)-E P^{s}\left(\frac{\bar{v}+d}{\bar{v}-d}\right)\right),
\end{aligned}
$$

given that the term in the first parenthesis has a stationary point at $d=0$ and the term in the second parenthesis is zero at $d=0$.

Once again, the "kinks" in the complete information model cause small changes to have large effects. In the incomplete information model, it has already been demonstrated that there are no first order effects of small changes in relative strength levels. The effects of the changes in the absolute strength of the two bidders exactly cancel out, at $d=0$. In this sense, small asymmetries are inconsequential.

\subsection{Concluding remarks}

The dominant assumption in the rent seeking literature is that information is complete. Among the advantages of such models are their analytical simplicity. Although the assumption of incomplete information is almost certainly more realistic, it is a challenge to build a tractable model which allows comparative statics. Such a model 
is presented in this paper. The model features a one-variable measure of strength, which means that comparative statics in the two models can easily be compared.

The results support the claim that incomplete information is important. The comparative statics are qualitatively different, in some cases diametrically opposed, in the two models. Put differently, in the space of all possible distribution functions, those that are degenerate give rise to different result than those that are not. Any amount of incomplete information changes the conclusions.

The main result is that ex ante asymmetries may cause more rent to be dissipated in the pursuit of the prize, contrary to the conclusion that obtains in a complete information model. Thus, asymmetry does not necessarily dampen competition.

\section{References}

[1] Alcalde, J. and Dahm, M., 2010, Rent seeking and rent dissipation: A neutrality result, Journal of Public Economics, 94:1-7.

[2] Amann, E. and Leininger, W., 1996, Asymmetric All-Pay Auctions with Incomplete Information: The Two-Player Case, Games and Economic Behavior, 14: $1-18$.

[3] Amegashie, J.A., 2006, Asymmetry and Collusion in Infinitely Repeated Contests, mimeo.

[4] Anderson, S.P., Goeree, J.K., and Holt, C.A., 1998, Rent Seeking with Bounded Rationality: An Analysis of the All-Pay Auction, Journal of Political Economy, 106 (4): 828-853.

[5] Baik, K.H., 1994, Effort Levels in Contests with Two Asymmetric Players, Southern Economic Journal, 61 (2): 367-378.

[6] Baye, M.R., Kovonock, D., and de Vries, C.G., 1993, Rigging the Lobbying Process: An Application of the All-Pay Auction, American Economic Review, 83 (1): 289-294.

[7] Baye, M.R., Kovonock, D., and de Vries, C.G., 1996, The all-pay auction with complete information, Economic Theory, 8: 291-305.

[8] Che, Y-K., and Gale, I., 2000, Difference-Form Contests and The Robustness of All-Pay Auctions, Games and Economic Behavior, 30: 22-43.

[9] Congleton, R.D., Hillman, A.L., and Konrad, K.A., 2008, 40 Years of Research on Rent Seeking, Vols. 1 and 2, Springer. 
[10] Fey, M., 2008, Rent-seeking contests with incomplete information, Public Choice, 135: 225-236.

[11] Fibich, G., Gavious, A., and Sela, A., 2004, Revenue equivalence in asymmetric auctions, Journal of Economic Theory, 115: 309-321.

[12] Hillman, A.L., and Riley, J.G., 1989, Politically Contestable Rents and Transfers, Economics and Politics, 1 (1): 17-39.

[13] Hurley, T.M. and Shogren, J.F., 1998, Effort levels in a Cournot Nash contest with asymmetric information, Journal of Public Economics, 69: 195-210.

[14] Kirkegaard, R., 2008, Comparative Statics and Welfare in Heterogenous All-Pay Auctions: Bribes, Caps, and Performance Thresholds, The B.E. Journal of Theoretical Economics, Vol. 8: Iss. 1 (Topics), Article 21.

[15] Kirkegaard, R., 2010a, Favoritism in Asymmetric Contests: Head Starts and Handicaps, mimeo.

[16] Kirkegaard, R., 2010b, Preferential Treatment may Hurt: Another Application of the All-Pay Auction, mimeo.

[17] Konrad, K.A., 2009, Strategy and Dynamics in Contests, Oxford University Press.

[18] Lebrun, B., 2009, Auctions with almost homogeneous bidders, Journal of Economic Theory, 144: 1341-1351.

[19] Malueg, D.A., and Yates, A.J., 2004, Rent Seeking with Private Values, Public Choice, 119 (1-2): 161-78.

[20] Myerson, R.B., 1981, Optimal Auction Design, Mathematics of Operations Research, 6: 58-73.

[21] Nti, K.O., 1999, Rent-seeking with asymmetric valuations, Public Choice, 98: 415-430.

[22] Parreiras, S.O. and Rubinchik, A., 2010, Contests with Three or More Heterogeneous Agents, Games and Economic Behavior, 68: 703-715.

[23] Tullock, G., 1967, The Welfare Costs of Tariffs, Monopolies, and Theft, Western Economic Journal, 5: 224-232.

[24] Tullock, G., 1980, Efficient Rent Seeking, in: James Buchanan, Roger Tollison, and Gordon Tullock (eds.), Towards a Theory of the Rent-Seeking Society, Texas A\&M University Press, 97-112.

[25] Wärneryd, K., 2003, Information in conflicts, Journal of Economic Theory, 110: 121-136. 